\title{
Proceso de registro y seguimiento de daños morfológicos en metal arqueológico mediante documentación 3D
}

\author{
Alba Fuentes-Porto, Elisa María Díaz-González, Manuel Drago Díaz-Alemán, Esteban Manuel \\ Amador-García
}

\begin{abstract}
Resumen: Gracias a la revolución tecnológica que vive el campo de la documentación del patrimonio vemos cómo los registros 3D se consolidan como recurso documental y se extienden con infinidad de propósitos, entre ellos los estudios morfométricos. Con la intención de contribuir en este campo, proponemos un método encaminado a la detección y cuantificación de cambios formales en bienes de naturaleza metálica, un tipo de material susceptible de sufrir daños físicos en un corto período de tiempo. La puesta en práctica de dicha metodología se ejemplifica a través del estudio efectuado sobre un casco de hierro del s. XVI, conservado en un avanzado estado de mineralización. Fue registrado antes y después de un préstamo temporal apoyándonos en dos sistemas de gran difusión en el campo de la digitalización 3D: el escaneado por luz estructurada y la fotogrametría. Finalmente, se compararon sus registros mediante la herramienta de análisis de datos 3D CloudCompare para detectar sus posibles diferencias.
\end{abstract}

Palabras clave: digitalización 3D, metales, análisis computacional, deterioro

\section{Processo de registro e monitoração de danos morfológicos em metais arqueológicos através da documentação 3D}

Resumo: Graças à revolução tecnológica que se vive no campo da documentação do património, vemos os registros 3D a consolidaremse como recursos documentais e a estenderem-se com finalidades infinitas, incluindo estudos morfométricos. Com o intuito de contribuir neste campo, propomos uma metodologia que visa detetar e quantificar as alterações formais em bens de natureza metálica, um tipo de material suscetível a danos físicos num curto espaço de tempo. A implementação desta metodologia é exemplificada através do estudo realizado num capacete de ferro do séc. XVI, conservado num avançado estado de mineralização. Foi registado antes e depois de um empréstimo temporário baseado em dois sistemas amplamente utilizados na área de digitalização 3D: digitalização estruturada de luz e fotogrametria. Finalmente, comparam-se os registos mediante a ferramenta de análise de dados 3D CloudCompare para detetar possíveis diferenças.

Palavras-chave: digitalização 3D, metais, análise computacional, deterioração

\section{Process of recording and tracking morphological damages in archaeological metal through 3D documentation}

Abstract:Thanks to the outstanding technological revolution that is taking place in the Cultural Heritage documentation field we see how 3D scanning has consolidated itself as a documentation resource and is expanding its functionality, such as the morphometric analysis. With the intention of contributing to this field we offer a methodology and workflow that aims to the detection and quantification of formal changes in metallic goods; a material that is susceptible to physical damage in a short span of time. The implementation of this methodology is exemplified through a study performed on an XVI century iron helmet, which has been preserved in an advanced state of mineralization. It was scanned before and after a temporary loan using two systems that are widely used in the field of 3D scanning: structured light scanning and photogrammetry. Both scannings were compared in the end with 3D CloudCompare software, a data analysis tool, to detect possible differences.

Keyword: 3D digitization, metals, computational analysis, damages 


\section{Introducción}

La revolución digital vivida en los últimos años ha traído consigo una importante renovación tecnológica en el campo de la documentación del patrimonio cultural. Gracias a ello, vemos cómo los registros 3D se consolidan como recurso documental y se extienden con infinidad de propósitos: documentación arqueológica, preservación y conservación digital, restauración virtual, museos multimedia, sistemas de información geográfica, y un largo etcétera (Remondino 2011).

Sus resultados realistas (en términos de precisión geométrica y radiométrica), combinados con medios informáticos para exploración de información geométrica cuentan también con un gran potencial analítico, en vías de desarrollo y normalización. Muchas de estas técnicas implican metodologías complejas que requieren conocimientos técnicos avanzados, pero otras resultan sencillas y son susceptibles de ser aprendidas por conservadores-restauradores con una capacitación básica en documentación 3D. Es el caso del estudio de diferencias geométricas entre registros, una función que en el campo del patrimonio se aplica con frecuencia a su control de calidad (Menna et al. 2016; Gagliolo et al. 2018; Grifoni et al. 2018). También encontramos ejemplos de su aplicabilidad para documentar cambios formales, bien como seguimiento de una intervención (Rahrig, Torge y Assets 2019), bien de procesos de deterioro (Pandey y Cather 2015; Ruiz et al. 2016).

Los metales, que a menudo presentan cierta maleabilidad o un estado de corrosión severo, conforman un tipo de materiales con una fragilidad matérica destacada y son susceptibles de sufrir daños físicos en un período corto de tiempo. Para contemplar su seguimiento nos valemos de fichas que recogen una descripción precisa de riesgos y alteraciones, además de registros fotográficos, mapas de daños o mediciones físicas (Díaz y García 2011: 6266). La incorporación de información morfométrica en el control de daños puede aportar información geométrica de precisión. En el presente artículo planteamos un método enfocado en la detección y cuantificación de daños formales en este tipo de bienes con la intención de contribuir a definir y ejemplificar sus posibilidades.

La puesta en práctica de dicha metodología contempla dos sistemas de digitalización 3D de gran difusión en patrimonio, el escaneado por luz estructurada y la fotogrametría. Se ejemplifica a través del estudio efectuado sobre un casco de hierro del s. XVI (Fragmento de morrión, 2012) depositado en el Museo Histórico Militar de Canarias (Tenerife) con motivo de su préstamo para participar en una exposición temporal. La pieza, de bordes irregulares y grosores reducidos, con unas dimensiones de $32 \times 38$ $\mathrm{cm}$, se conserva parcialmente en un avanzado estado de mineralización [figura 1].

\section{Metodología}

Gracias a la destacada resolución y precisión que han alcanzado las técnicas de escaneado 3D y fotogrametría, desde hace una década campos de estudio como el de la geomorfología recurren al análisis de registros 3D para seguir la evolución de la superficie terrestre a través de procesos dinámicos como deslizamientos, desprendimientos o procesos erosivos (Lague, Brodu y Leroux 2013). Contar con una secuencia de registros 3D a lo largo de un intervalo de tiempo determinado también ofrece un gran potencial analítico para detectar y medir la evolución de cambios físicos en cualquier tipo de patrimonio material. Interesantes ejemplos de aplicabilidad los encontramos en el estudio de grabados rupestres (Ruiz et al. 2016) y pinturas murales (Pandey y Cather 2015), ambos patrimonio inmueble.

A la hora de trasladar este tipo de análisis morfométricos a piezas metálicas debemos contar con información geométrica de precisión. Para ello se requiere cuidar la

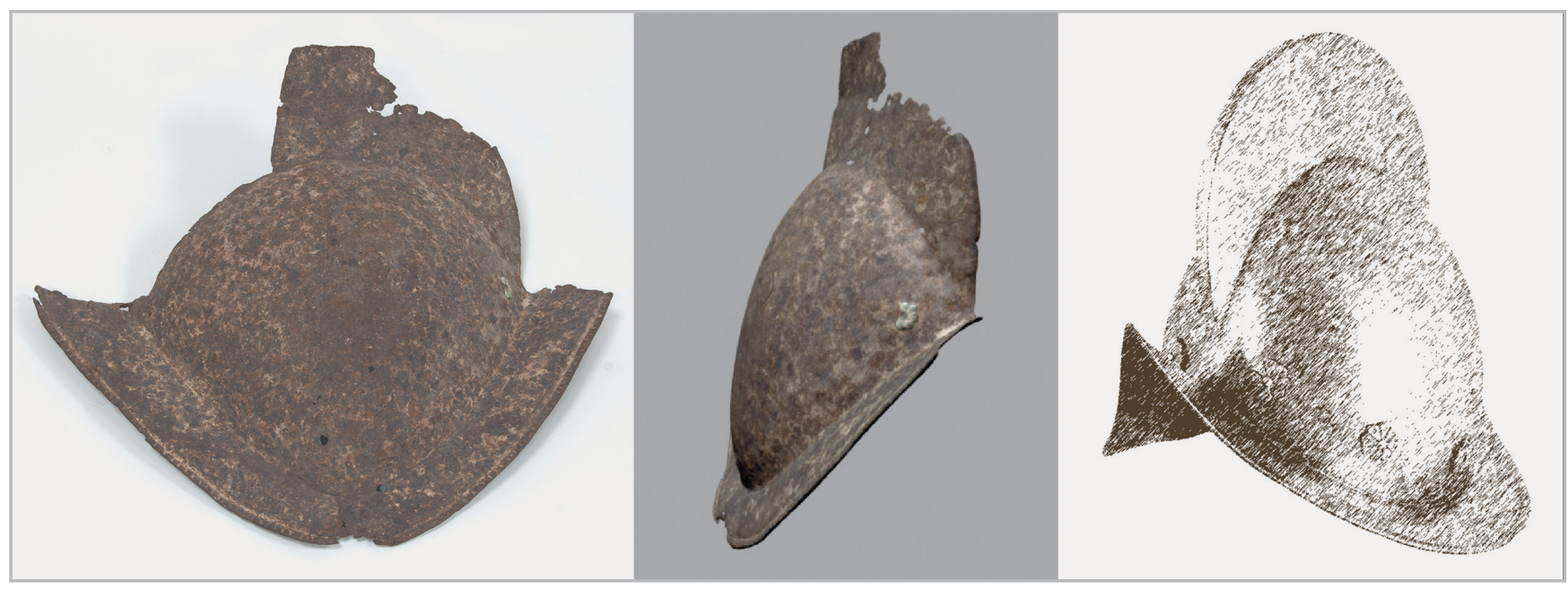

Figura 1.- Morrión español del S.XVI, depositado en el Museo Histórico Militar de Canarias. Autor: Alba Fuentes-Porto. 
metodología de forma que se minimicen las posibles diferencias entre digitalizaciones periódicas, y se alcancen valores de resolución adecuados al tamaño y detalle formal de cada tipo de pieza. En este sentido, definir un protocolo de documentación estricto permitirá la reproductibilidad tanto de las condiciones de captura como del procesamiento de los datos. Además, ayudará a adaptar los parámetros del registro a las peculiaridades de cada pieza y a maximizar la capacidad de resolución de los dispositivos de captura. Es la resolución la que va a condicionar nuestra capacidad interpretativa, por lo que, siempre que sea posible, es recomendable alcanzar valores submilimétricos que posibiliten la detección de daños estructurales como fragmentaciones, fracturas o incluso delaminaciones de los estratos de corrosión.

\section{—Adquisición de información geométrica}

El escaneo 3D mediante luz estructurada y la fotogrametría son dos técnicas empleadas con frecuencia en el registro y la documentación del patrimonio. Ofrecen una excelente precisión geométrica y permiten generar modelos 3D de alta calidad de piezas de pequeña y mediana escala (Bruno et al. 2013; Gil-Melitón y Lerma 2018; Morita y Bilmes 2018; Marshall et al. 2019).

El escáner de luz estructurada es un dispositivo capaz de capturar la forma de un objeto mediante un sistema de medición óptica que proyecta patrones de luz y aborda la medición trigonométrica de sus distorsiones para calcular la profundidad de los puntos inspeccionados (Pieraccini, Guidi y Atzeni 2001). Aporta medidas reales de la superficie del objeto a escala 1:1, además de una gran resolución y precisión 3D. En los dispositivos más extendidos dentro de nuestro campo éstas oscilan entre los $0.1 / 0.5 \mathrm{~mm}$ de resolución $3 \mathrm{D}$ o distancia entre puntos de muestreo, y los 0.05/0.1 mm de precisión de puntos (Di Angelo et al. 2018; Artec Group 2020). También existen escáneres metrológicos de alta precisión, diseñados para el registro de piezas pequeñas, que llegan a alcanzar las 30 micras $(0.03 \mathrm{~mm})$ y 10 micras $(0.01 \mathrm{~mm})$ respectivamente.

Sin embargo, al tratarse de un método óptico, las superficies reflectantes o transparentes plantean dificultades a la hora de ser registradas, ya que generan efectos de luz que pueden superponerse al de los patrones imposibilitando su lectura. Las formas cóncavas, los grosores reducidos y las zonas negras o muy oscuras también pueden ocasionar vacíos de información (Gupta et al. 2011; Graciano et al. 2017). Estas limitaciones van a afectar a la hora de registrar piezas que conserven su brillo metálico, que presenten tonalidades oscuras o que cuenten con incrustaciones de cristales o gemas.

La fotogrametría se basa en sofisticados algoritmos de coincidencia de imágenes que utilizan fotografías superpuestas, adquiridas desde múltiples puntos de vista, para reconstruir la geometría tridimensional de un objeto o superficie (Aicardi et al. 2018; Vanneschi et al. 2019: 5). La resolución de los modelos fotogramétricos viene determinada por el GSD (Ground Sampling Distance), que define cuánto mide un píxel sobre el objeto. Es directamente proporcional a la distancia de registro, al tamaño y resolución del sensor de la cámara, y a la distancia focal empleada (Verhoeven 2018). En la fotogrametría de objeto cercano se pueden alcanzar resoluciones submilimétricas. Para ello es necesario adquirir un número elevado de fotografías con alta redundancia o solape (Guidi et al. 2015; Sapirstein 2018), además de acercarse lo máximo posible al objeto documentado (Verhoeven 2019). En la actualidad contamos con guías de buenas prácticas para el registro fotogramétrico del patrimonio, publicadas por instituciones de referencia, que recogen flujos y parámetros de trabajo para una documentación geométrica de calidad (Eulac Museums 2013; Historic England 2017).

No aporta medidas reales, siendo necesario tomar mediciones físicas de referencia para proyectarlas durante el procesado de los datos. Sin embargo, ofrece una mayor resolución de imagen que las técnicas de escaneado y permite implementar procesos de calibración cromática con vistas a obtener un registro preciso del color (Ruiz y Pereira 2014; Molada-Tebar, Marqués-Mateu y Lerma 2019). A esto se le suma una considerable accesibilidad económica y facilidad de implementación, conjunto de cualidades que avala a la fotogrametría como una alternativa sólida al escaneo 3D.

Los efectos de luz que generan las superficies reflectantes y los grosores reducidos siguen causando complicaciones y produciendo ruido en las lecturas espaciales, pero la captura de un número elevado de fotografías solventa este problema y aporta un píxel estable y seguro a la hora de construir un correcto modelo 3D (Gil-Melitón y Lerma 2018).

En el caso práctico que se presenta se utilizó como dispositivo de luz estructurada un modelo Artec Eva (Artec3D), muy extendido en la documentación 3D del patrimonio. Ofrece una resolución de $0,5 \mathrm{~mm}$ y una precisión de 0,1 $\mathrm{mm}$.

Para el levantamiento fotogramétrico se empleó una cámara DSLR con un tamaño de píxel de $4.29 \mu \mathrm{m}$ (modelo Canon EOS 700D). Las capturas se realizaron a una distancia del objeto de $132 \mathrm{~cm}$, con una focal fija de $47 \mathrm{~mm}$, un diafragma f11 e ISO 100. Fueron tomadas 70 fotografías de cada lado de la pieza, con una distribución en bucle facilitada por una mesa giratoria, formato RAW y máxima resolución. El valor GSD alcanzado fue de $0.12 \mathrm{~mm} /$ pix, lo que supone que cada píxel de las imágenes representa $0.12 \mathrm{~mm}$ de la pieza registrada.

Las formas semiesféricas y grosores reducidos del casco suelen dificultar las labores de alineado en ambas técnicas. Para contrarrestarlo, durante el proceso de escaneado se 
dispusieron un conjunto de dianas. Además, el área de la cresta nos permitió digitalizar de forma simultánea zonas de su anverso y su reverso, lo que proporcionó datos con superficies parciales en ambos lados e hizo posible registrar su espesor. Sin embargo, bordes y lagunas no se lograron registrar adecuadamente debido a su irregularidad y finura. En el levantamiento fotogramétrico la pieza se colocó sobre un pequeño pedestal para potenciar la visibilidad de sus dos caras [figura 2].

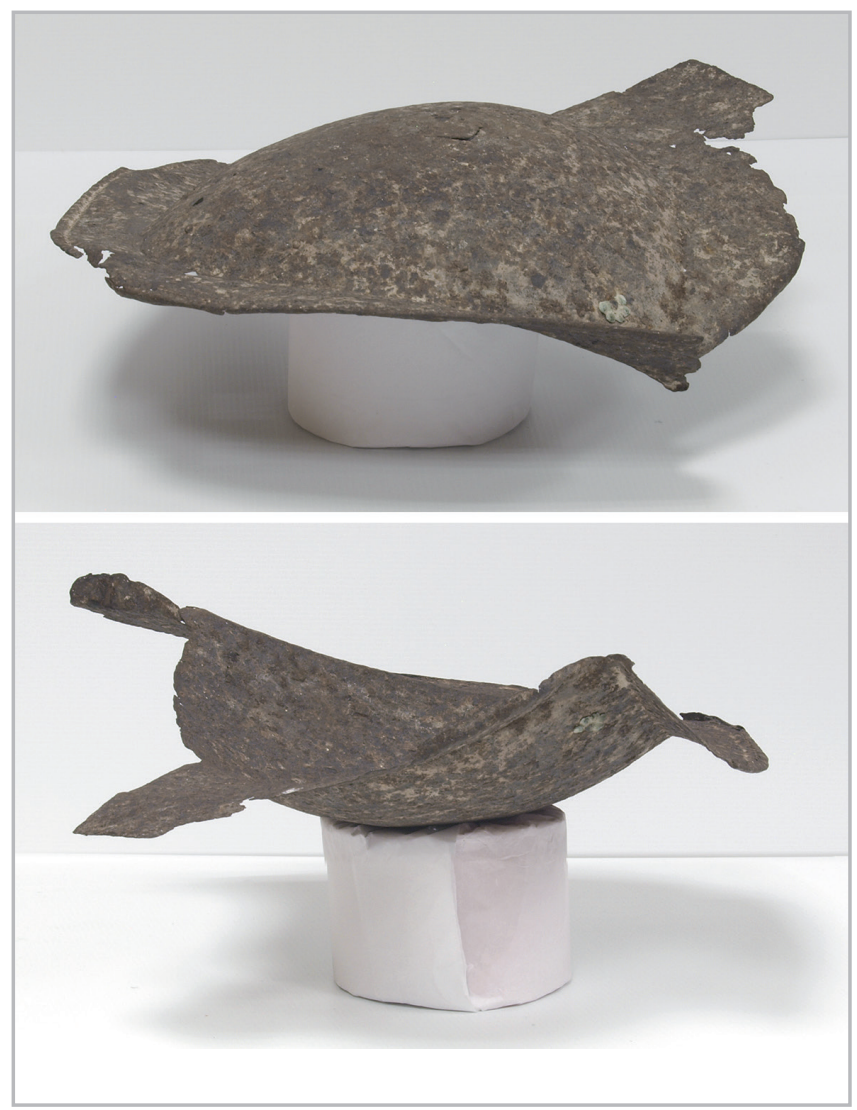

Figura 2.- Disposición de ambos lados de la pieza en el registro fotogramétrico. Autor: Alba Fuentes-Porto.

\section{—Procesado de registros 3D}

A la hora de emplear modelos 3D con fines analíticos, conviene configurar los parámetros de edición a máxima calidad para maximizar su precisión y resolución geométrica. Mientras que los modelos de mallas texturizados son los más adecuados para proporcionar una representación realista de la superficie, las nubes de puntos se adecúan mejor a los análisis morfométricos, ya que eluden la simplificación de zonas, se reduce el procesado de los datos y ayudan a acelerarlo durante las tareas de optimización y análisis. Un aspecto que se debe cuidar es la correcta ubicación de las normales, vectores perpendiculares a la geometría registrada que determinan la orientación de la superficie. Si estas no se corresponden con la realidad física del objeto generarán errores de lectura.
Los sistemas de escaneado 3D cuentan con su propio software de procesado de datos y suelen presentar flujos de trabajo típicos: escaneo o adquisición de datos, registro y alineado de escaneos, eliminación de ruido e información superflua, generación y diezmado de malla, generación y optimización de texturas, exportación en diferentes formatos, visualización e incluso realización de mediciones sencillas.

Como software de procesado fotogramétrico disponemos de varias opciones con un amplio abanico de prestaciones y precios, existiendo también herramientas gratuitas (Kingsland 2019). Hoy en día, Agisoft Metashape (Agisoft) es una de las más empleadas en la documentación 3D del patrimonio cultural y sus evaluaciones afirman su fiabilidad y reproducibilidad en reconstrucciones digitales (Kingsland 2019). Su flujo de trabajo estándar contempla la calibración de cámara, alineado de fotografías y generación de puntos de paso, la creación de mapas de profundidad y de nube de puntos densa, la eliminación de información superflua y filtrado de ruido, el mallado, el alineado y unión de bloques, el texturizado y la exportación.

En ambos casos, el software de procesado se actualiza de forma constante incorporando mejoras en sus algoritmos y en sus prestaciones. Por ello, conviene guardar los archivos en bruto y reprocesarlos a la hora de hacer seguimientos a largo plazo.

En el caso que se presenta, los registros realizados por escaneado mediante luz estructurada se procesaron utilizando el software Artec Studio Professional V.10 (Artec3D) con los valores predeterminados para una edición de alta calidad [figura 7].

Con vistas a reflejar flujos de trabajo actuales, los registros fotográficos fueron reprocesados con la versión de Agisoft Metashape V.1.6.4 Standard Edition (Agisoft), lanzada en 2020. Como paso previo se realizó un revelado RAW contemplando su calibración cromática. Para ello se ajustó la curva tonal con la ayuda de una carta de color X-Rite ColorChecker Passport (X-Rite/Pantone) siguiendo la metodología detallada por Santos (2014), y se exportaron a formato JPG. A continuación, en Adobe Photoshop V.22.0 (Adobe) se enmascararon las imágenes en el canal alfa con el fin de focalizar la detección de puntos de paso y puntos clave sobre el objeto. A continuación, los archivos fueron importados al software de procesado, en el que se realizó un flujo de trabajo tipo [figura 7].

Agisoft Metashape (Agisoft) incorpora la opción de estimar el nivel de confianza de los puntos a la hora de generar la nube de puntos densa. Esto, unido a las herramientas de filtrado por nivel de confianza, permitió eliminar el ruido con destacada eficiencia y alcanzar registros más depurados y coherentes [figura 3]. 

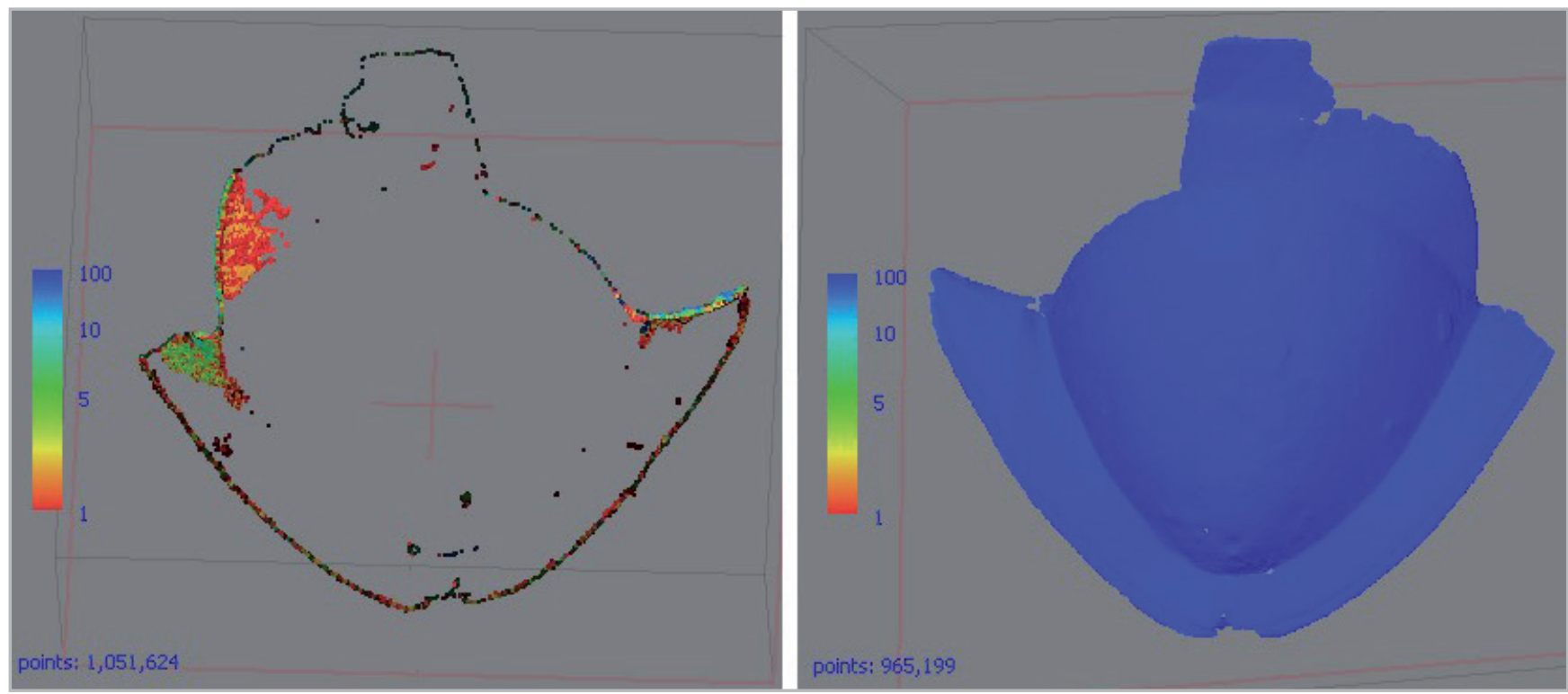

Figura 3.- Ejemplo del filtrado por nivel de confianza de uno de los registros fotogramétricos. Izda.) Selección de valores inferiores a 20. Dcha.) Nube de puntos tras su eliminación. Autor: Alba Fuentes-Porto.

En todos los registros, las nubes de puntos fueron exportadas en formato PLY (.ply), tipo de archivo que guarda las coordenadas geométricas, color y normales de cada uno de los vértices. Por último, se comprobó la coherencia geométrica de los registros mediante la constatación de la correcta orientación de sus normales. Esto se hizo en MeshLab v.2020.07 (MeshLab), software gratuito que cuenta con un interesante conjunto de herramientas para limpiar, reparar e inspeccionar datos 3D. El proceso consistió en dos sencillos pasos, cuyos comandos se facilitan en inglés por ser el único idioma del software:

1. Filters > Normals, Curvatures and Orientation > Compute normals for point sets

2. Render $>$ Show Normal [figura 4].

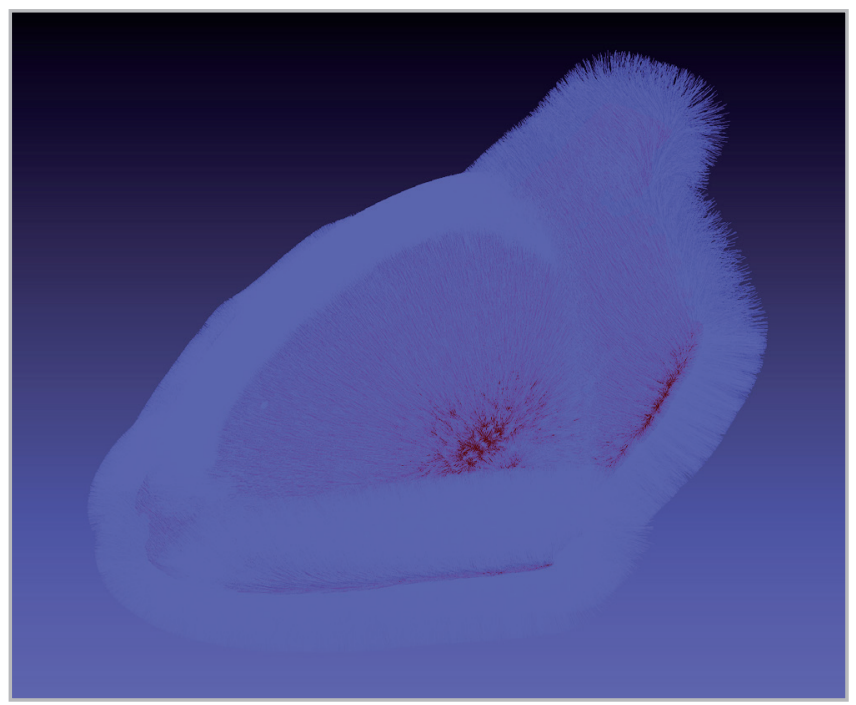

Figura 4.- Visualización de la orientación y longitud de las normales en MeshLab. Autor: Alba Fuentes-Porto.
Si no figuran las normales o están mal orientadas podemos corregirlas, asegurándonos que guardamos estos atributos de forma correcta al exportar el archivo:

3. Filters $>$ Normals, Curvatures and Orientation $>$ Compute normals for point sets.

\section{—Análisis morfométrico}

CloudCompare V.2.11.3 (Proyecto CloudCompare) es una aplicación gratuita y de código abierto dirigida al procesado y análisis geométrico de modelos 3D (Girardeau-Montaut 2006). Cuenta con tres herramientas para detectar las diferencias y computar las distancias existentes entre dos registros:

- Cloud to Mesh Distance (C2M) computa las distancias entre una nube de puntos y una malla (Girardeau-Montaut 2015b).

- Cloud to Cloud Distance (C2C) computa las distancias entre dos nubes de puntos. Para cada punto de la nube comparada, el software busca el punto más cercano en la nube de referencia y calcula su distancia (GirardeauMontaut 2015a).

- Multiscale Model to Model Cloud Comparison (M3C2) también opera directamente sobre nubes de puntos, pero la distancia se calcula a lo largo de la dirección de las normales (Lague, Brodu y Leroux 2013; Girardeau-Montaut 2019).

Autores del ámbito de la teledetección consideran que $\mathrm{M} 3 \mathrm{C} 2$ es la herramienta más adecuada para resolver la detección de pequeños cambios por hacer un tratamiento matizado de los datos (Barnhart y Crosby 2013: 2828). Sin embargo, la configuración de sus parámetros requiere conocimientos 
técnicos avanzados; mientras que $\mathrm{C} 2 \mathrm{C}$ ofrece un entorno más sencillo e intuitivo, a la vez que proporciona "distancias verdaderas" siempre que la nube de referencia cuente con una densidad suficiente. Ambas características nos llevan a estimar esta opción como la alternativa más adecuada para un uso generalizado y es la que se recoge a continuación.

El punto de partida para analizar una pareja de registros 3D consiste en hacer que pasen a ocupar un mismo espacio, a partir del cual poder computar las diferencias. Es un procedimiento indispensable para no generar falsos resultados por discordancias en la orientación de ambos modelos. Se consigue mediante su alineado y registro, siendo conveniente tomar como referencia siempre la nube de puntos más densa:

1. Tools $>$ Registration $>$ Match bounding-box centers.

2. Tools $>$ Registration $>$ Align (por selección de pares de puntos).

3. Tools > Registration > Fine registration. Este proceso permite ajustar la escala entre un par de modelos, opción que conviene activar en los fotogramétricos para eliminar cualquier diferencia que pudiera ocasionar su escalado manual.

Una vez conseguida la coherencia geométrica se procede a calcular las diferencias morfométricas. El propio software estima los parámetros que mejor se adecúan al cálculo, por lo que el comando se puede ejecutar de forma directa:

\section{Tools $>$ Distances $>$ Cloud/Cloud Dist.}

Para el análisis de los posibles cambios originados en el morrión se usó el registro de vuelta tanto en el escaneado como en la fotogrametría, y se aplicó la herramienta con los parámetros preestablecidos [figuras 5 y 6].

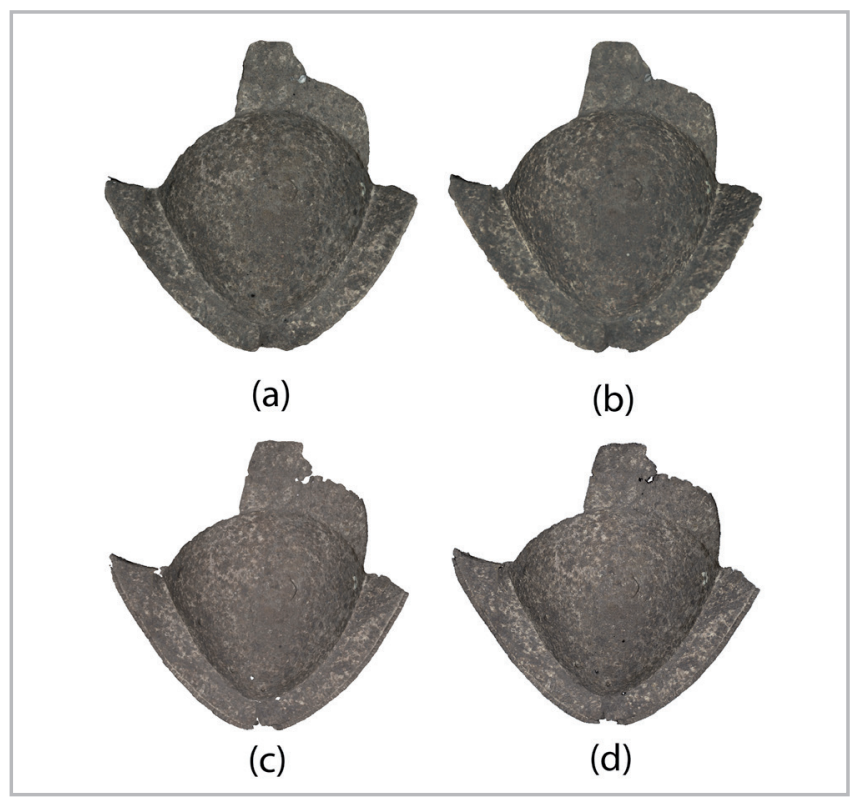

Figura 5.- Registros 3D de salida (izda.) y entrada (dcha.) adquiridos mediante escaneado por luz estructurada $(a, b)$ y fotogrametría (c,d). Autor: Alba Fuentes-Porto.

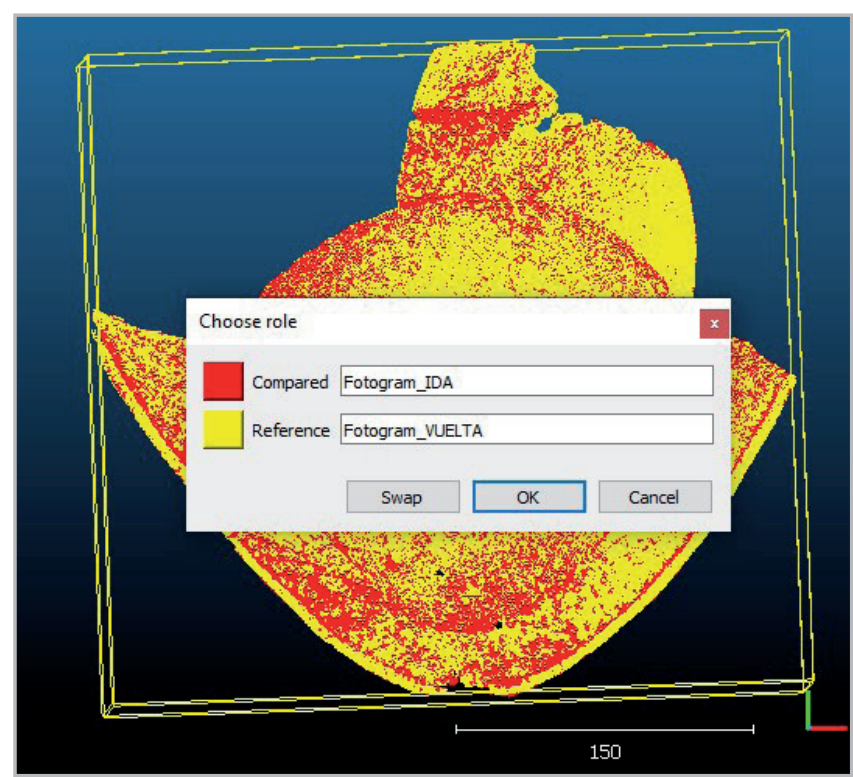

Figura 6.- Selección de la nube de referencia tras el alineado de modelos fotogramétricos. Autor: Alba Fuentes-Porto.

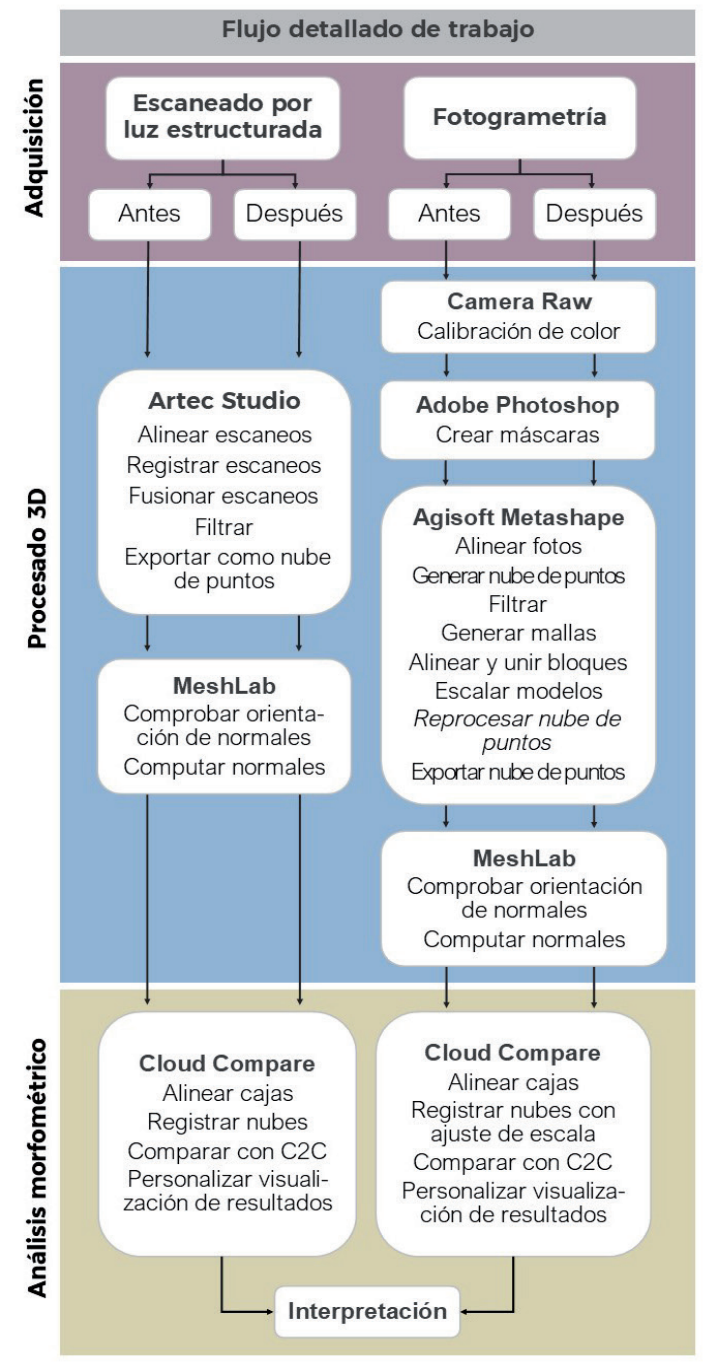

Figura 7.- Flujo de trabajo seguido: adquisición de información geométrica, procesado de datos 3D, y análisis morfométrico. Autor: Alba Fuentes-Porto. 
Realizado el cálculo, se proyectan diferencias geométricas sobre la nube analizada, que son resaltadas por medio de una codificación cromática. Su apariencia es configurable a través de la ventana de propiedades. También cuenta con la posibilidad de añadir una escala numérica con los valores cuantitativos de las distancias, y un histograma. Este gráfico nos permite estudiar su tendencia y distribución con más detalle. Ambos elementos se pueden activar con los comandos:

\section{Display $>$ Active scalar field $>$ Toggle color scale 6. Edit > Scalar Fields > Show Histogram}

La capacidad de análisis dependerá de la resolución de los registros. A la hora de interpretar los resultados será necesario buscar tendencias locales. Es probable que los resultados abarquen valores de distancias elevados, pero suelen deberse a puntos de ruido aislados.

\section{Resultados}

Loscambiossignificativosquesedetectan entrelos registros adquiridos con el escáner de luz estructurada se disponen exclusivamente a lo largo del perímetro de la pieza, zona que no había sido registrada convenientemente y carece de datos sólidos. Si recurrimos al histograma del análisis

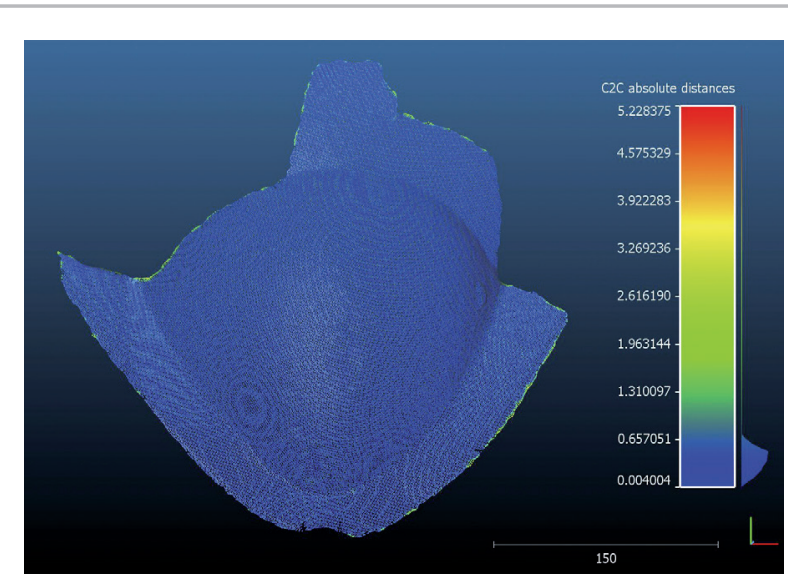

(a)

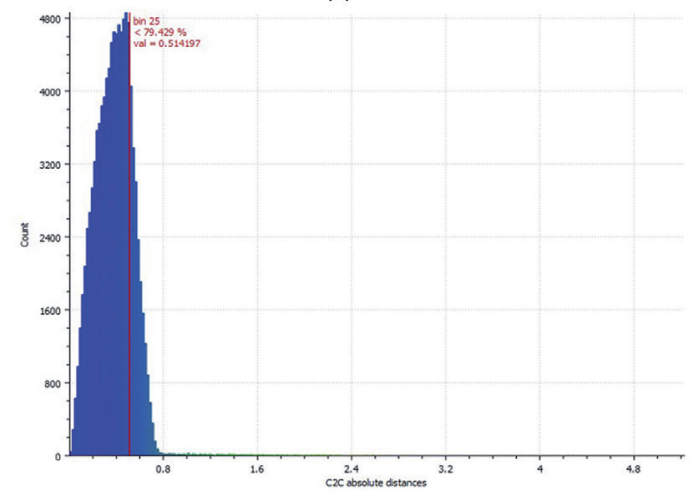

(b)

Figura 8.- Mapa de diferencias e histograma. Reflejan los resultados del análisis sobre los escaneados. Autor: Alba Fuentes-Porto. morfométrico, podemos observar que estas diferencias se concentran, en pequeñas tendencias locales de cierta entidad, entre los $0.8 \mathrm{~mm}$ y los $2 \mathrm{~mm}$. El interior de la pieza no registra ningún cambio significativo, mientras que la curva de distribución muestra una tendencia homogénea hasta los $0.8 \mathrm{~mm}$, valores que nos llevan a determinar que no se han producido ganancias o pérdidas de material por encima de estas medidas [figura 8].

Los registros fotogramétricos no alertan de ningún cambio, incluido el perímetro de la pieza, registrado de forma correcta. Su curva de distribución muestra una tendencia homogénea hasta los $0.4 \mathrm{~mm}$. La ausencia de tendencias locales en las diferencias halladas por encima de los $0.4 \mathrm{~mm}$ subraya que no se ha detectado ningún cambio significativo [figura 9].

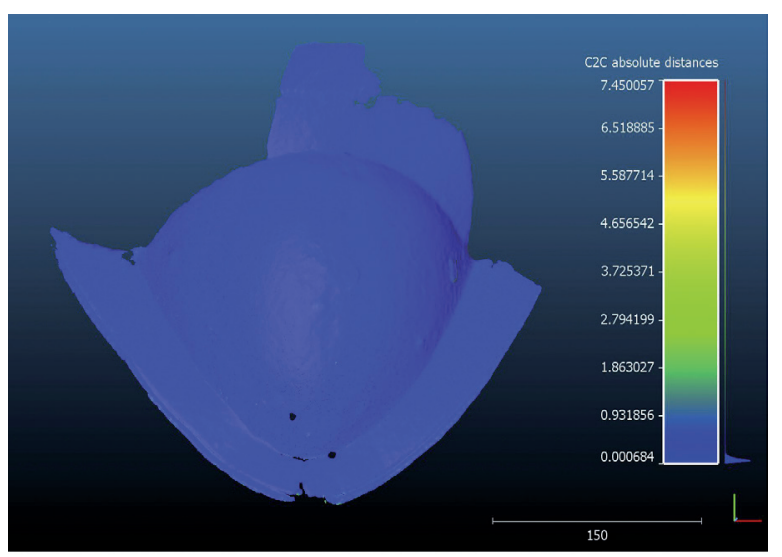

(a)

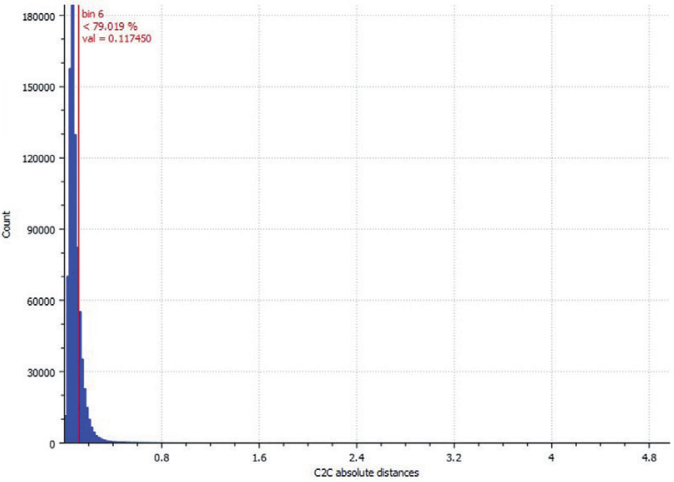

(b)

Figura 9.- Mapa de diferencias e histograma. Reflejan los resultados del análisis sobre los registros fotogramétricos. Autor: Alba Fuentes-Porto.

\section{Discusión}

El caso práctico presentado refleja cómo los modelos 3D y el análisis espacial pueden contribuir a optimizar el seguimiento formal de piezas metálicas, ya que aportan documentación geométrica de precisión dotada de información cuantitativa. Además, hoy en día, ciertos procesadores y lectores de texto contemplan la inserción de este tipo de imágenes. Esto nos permite integrarlas en 
nuestros informes técnicos e interactuar con ellas, lo que salva las restricciones de perspectiva común a los registros bidimensionales e incluso nos capacita para acometer mediciones sencillas.

Nuestra capacidad de detección dependerá de la resolución de los registros. Está condicionada por la propia resolución de nuestros dispositivos de captura, el grado de dificultad de la pieza y el coste en tiempo del que se disponga. Cuando nos enfrentamos al registro de piezas metálicas de tamaño medio, será conveniente alcanzar resoluciones submilimétricas que puedan registrarnos deformaciones, fragmentaciones, fracturas 0 incluso delaminaciones. Cambios de volumen más sutiles, como un incremento paulatino debido a procesos corrosivos o variaciones dimensionales producidas por diferencias de temperatura en el momento de los registros, requieren una resolución de micras y escáneres metrológicos de alta precisión; siendo una vía interesante de investigación.

A la hora de digitalizar esta pieza de hierro de tamaño medio, textura homogénea, formas cóncavas y grosores finos, el escáner por luz estructurada empleado nos ofreció una resolución de $0.5 \mathrm{~mm}$, suficiente para detectar daños estructurales submilimétricos, además de información métrica a escala 1:1 y de gran precisión, con nubes de puntos homogéneas y con poco ruido. Sin embargo, los bordes y lagunas de la pieza no se registraron convenientemente debido a la irregularidad y finura del objeto.

Si nos valemos de la propia herramienta C2C para comparar ambos registros podemos ver la extensión exacta de las zonas no registradas por el escáner de luz estructurada [figura 10].

La fotogrametría sí logró documentar estos detalles. La precisión de sus registros dependió en gran medida del software de procesado. Así, entre el 2018 y 2019, año de

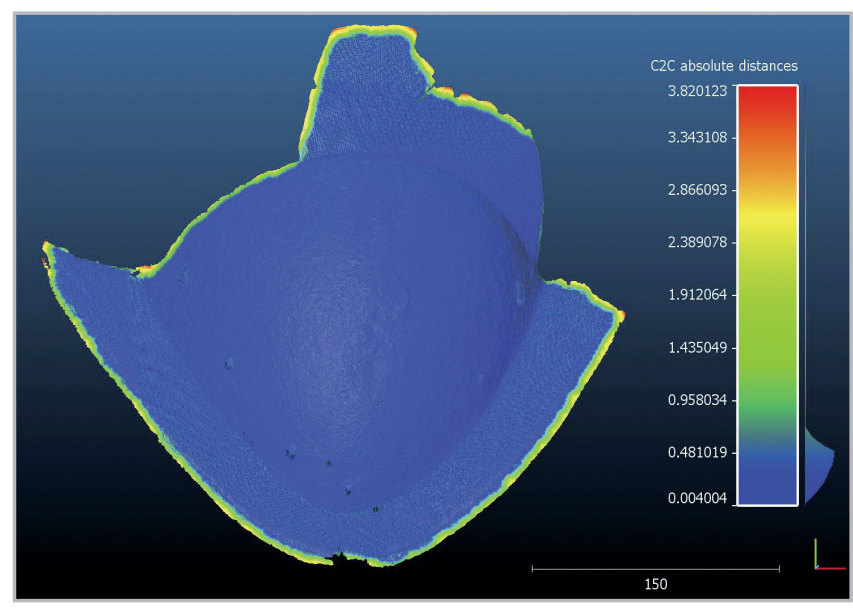

Figura 10.- Comparativa entre la geometría del escaneado de luz estructurada y el modelo fotogramétrico. Autor: Alba Fuentes-Porto. desarrollo del proyecto, una limpieza basada en métodos manuales no nos facilitó un depurado eficiente de ruido (Fuentes-Porto, Díaz-Aleman y Díaz-González 2020), labor que hizo posible la incorporación de las herramientas de estimación y filtrado por nivel de confianza de puntos que incorporó Agisoft Metashape (Agisoft). También se alcanzó una destacada resolución, definida por un GSD de 0.12 $\mathrm{mm} /$ pix.

Es innegable que los registros 3D son, en la actualidad, el mejor método de obtención de mediciones muy precisas. Esto no nos exime de que, en función de las características formales de cada pieza, los recursos tecnológicos y los tiempos de trabajo de los que dispongamos, podamos encontrarnos con limitaciones técnicas que deriven en lagunas documentales o precisiones inferiores a las esperadas. En ese caso, siempre debemos ajustarnos a los datos sólidos, que enriquecerán nuestra capacidad interpretativa por limitados que sean.

\section{Conclusiones}

El desarrollo y los resultados de este trabajo experimental subrayan la aplicabilidad de las técnicas geomáticas en el seguimiento y monitorización formal de piezas metálicas. Siguiendo una metodología cuidada, registros adquiridos mediante escaneado de luz estructurada o fotogrametría han posibilitado análisis geométricos de precisión submilimétrica capaces de cuantificar cambios volumétricos a lo largo de un intervalo temporal. La incorporación de información morfométrica en los protocolos de control de daños contribuye a minimizar la subjetividad de valoraciones técnicas que hasta la fecha se respaldaban en la comparación de registros fotográficos, mapas de daños o mediciones físicas. La accesibilidad y sencillez de la metodología favorece la normalización de este tipo de procedimientos, pero es necesario que toda interpretación se ajuste a datos sólidos.

\section{Agradecimientos}

Los autores agradecen el apoyo brindado por el Museo Histórico Militar de Canarias y el Servicio General de Apoyo a la Investigación (SEGAI) de la Universidad de La Laguna a través de sus laboratorios de Microscopía Electrónica y Difracción de Rayos X.

\section{Referencias}

AICARDI, I., CHIABRANDO, F., MARIA LINGUA, A. NOARDO, F. (2018) "Recent trends in cultural heritage 3D survey: The photogrammetric computer vision approach", Journal of Cultural Heritage, 32: 257-266. https://doi.org/doi: 10.1016/j. culher.2017.11.006.

DI ANGELO, L., DI STEFANO, P., FRATOCCHI, L., MARZOLA, A. (2018) 
"An AHP-based method for choosing the best 3D scanner for cultural heritage applications", Journal of Cultural Heritage, 34: 109-115. https://doi.org/10.1016/j.culher.2018.03.026.

\section{ARTEC GROUP (2020) Escáneres 3D portátiles, Artec 3D. Disponible en: $\quad$ https://www.artec3d.com/es/portable-3d-scanners [consulta: 28/12/2020].}

BARNHART, T.B. Y CROSBY, B.T. (2013) "Comparing two methods of surface change detection on an evolving thermokarst using high-temporal-frequency terrestrial laser scanning, Selawik River, Alaska", Remote Sensing, 5(6): 2813-2837. https://doi.org/10.3390/ rs5062813.

BRUNO, F., GALLO, A., DE FILIPPO, F., MUZZUPAPPA, M., DAVIDDE PETRIAGGI, B., CAPUTO, P. (2013) "3D documentation and monitoring of the experimental cleaning operations in the underwater archaeological site of Baia (Italy)", Proceedings of the DigitalHeritage 2013, 105-112. https://doi.org/10.1109/ DigitalHeritage.2013.6743719.

DÍAZ MARTÍNEZ, S. Y GARCÍA ALONSO, E. (2011) Técnicas metodológicas aplicadas a la conservación-restauración del patrimonio metálico. Madrid: Ministerio de Cultura, Gobierno de España.

EULAC MUSEUMS (2013) "Manual para la digitalización 3D y tecnologías esféricas.", Journal of Chemical Information and Modeling, 14. https://doi.org/10.1017/CBO9781107415324.004.

FUENTES-PORTO, A., DÍAZ-ALEMAN, D. Y DÍAZ-GONZÁLEZ, E. (2020) "3D Recording and Point Cloud Analysis for Detecting and Tracking Morphological Deterioration in Archaeological Metals", en Innovation in Information Systems and Technologies to Support Learning Research, 362-367. https://doi.org/10.1007/978-3-03036778-7 40.

GAGLIOLO, S., AUSONIO, E., FEDERICI, B., FERRANDO, I., PASSONI, D., SGUERSO, D. (2018) "3D cultural heritage documentation: A comparison between different photogrammetric software and their products", International Archives of the Photogrammetry, Remote Sensing and Spatial Information Sciences - ISPRS Archives, 42(2): 347-354. https://doi.org/10.5194/isprs-archivesXLII-2-347-2018.

GIL-MELITÓN, M. Y LERMA, J. L. (2018) "Patrimonio histórico militar: digitalización 3D de la espada nazarí atribuida a Ali Atar", Virtual Archaeology Review, 10(20): 52. https://doi.org/10.4995/ var.2019.10028.

GIRARDEAU-MONTAUT, D. (2006) Détection de changement sur des données géométriques tridimensionnelles. ENST.

GIRARDEAU-MONTAUT, D. (2015a) Cloud-to-Cloud Distance, CloudCompare Wiki. Disponible en: https://www.cloudcompare. org/doc/wiki/index.php?title=Cloud-to-Cloud Distance [consulta: 30/12/2020].

GIRARDEAU-MONTAUT, D. (2015b) Cloud-to-Mesh Distance,
CloudCompare Wiki. Disponible en: https://www.cloudcompare. org/doc/wiki/index.php?title=Cloud-to-Mesh Distance [consulta: 30/12/2020].

GIRARDEAU-MONTAUT, D. (2019) M3C2 (plugin), CloudCompare Wiki. Disponible en: https://www.cloudcompare.org/doc/wiki/ index.php?title=M3C2 (plugin) [consulta: 30/12/2020].

GRACIANO, A., ORTEGA, L., SEGURA, R.J., FEITO, F.R. (2017) "Digitization of religious artifacts with a structured light scanner", Virtual Archaeology Review, 8(17): 49-55. https://doi.org/10.4995/ var.2016.4650.

GRIFONI, E., LEGNAIOLI, S., NIERI, P., CAMPANELLA, B., LORENZETTI, G., PAGNOTTA, S., POGGIALINI, F., PALLESCHI, V. (2018) "Construction and comparison of 3D multi-source multi-band models for cultural heritage applications", Journal of Cultural Heritage, 34: 261-267. https://doi.org/10.1016/j. culher.2018.04.014.

GUIDI, G., MICOLI, L.L., GONIZZI, S., BRENNAN, M., FRISCHER, B. (2015) "Image-based 3D capture of cultural heritage artifacts: An experimental study about 3D data quality", en 2015 Digital Heritage International Congress, Digital Heritage 2015. Institute of Electrical and Electronics Engineers Inc., 321-324. https://doi. org/10.1109/DigitalHeritage.2015.7419514.

GUPTA, M., AGRAWAL, A., VEERARAGHAVAN, A., NARASIMHAN, S.G. (2011) “Structured light 3D scanning in the presence of global illumination", Proceedings of the IEEE Computer Society Conference on Computer Vision and Pattern Recognition, 713-720. https://doi. org/10.1109/CVPR.2011.5995321.

HISTORIC ENGLAND (2017) Photogrammetric Applications for Cultural Heritage: Guidance for Good Practice. Londres: Historic England. https://doi.org/10.1212/01.WNL.0000132885.83350.45.

KINGSLAND, K. (2019)“A Comparative Analysis of Two Commercial Digital Photogrammetry Software for Cultural Heritage Applications", en Lecture Notes in Computer Science, 70-80. https:// doi.org/10.1007/978-3-030-30754-7 8.

LAGUE, D., BRODU, N.Y LEROUX, J. (2013) “Accurate 3D comparison of complex topography with terrestrial laser scanner : application to the Rangitikei canyon (N-Z)", ISPRS Journal of Photogrammetry and Remote Sensing, 82: 10-26. https://doi.org/10.1016/j. isprsjprs.2013.04.009.

MARSHALL, M. E., JOHNSON, A.A., SUMMERSKILL, S. J., BAIRD, Q., ESTEBAN, E. (2019) "Automating photogrammetry for the 3D digitisation of small artefact collections", en International Archives of the Photogrammetry, Remote Sensing and Spatial Information Sciences - ISPRS Archives. International Society for Photogrammetry and Remote Sensing, 751-757. https://doi. org/10.5194/isprs-archives-XLII-2-W15-751-2019.

MENNA, F., NOCERINO, E., REMONDINO, F., DELLEPIANE, M., CALLIERI, M., SCOPIGNO, R. (2016) "3D digitization of an heritage masterpiece-a critical analysis on quality assessment", 
International Archives of the Photogrammetry, Remote Sensing and Spatial Information Sciences - ISPRS Archives, XLI-B5, 675-683. https://doi.org/10.5194/isprsarchives-XLI-B5-675-2016.

MOLADA-TEBAR, A., MARQUÉS-MATEU Y LERMA, J. L. (2019) "Correct use of color for cultural heritage documentation", en ISPRS Annals of the Photogrammetry, Remote Sensing and Spatial Information Sciences, 107-113. https://doi.org/10.5194/isprsannals-IV-2-W6-107-2019.

MORITA, M. Y BILMES, G. (2018) "Applications of low-cost 3D imaging techniques for the documentation of heritage objects", Optica Pura y Aplicada, 51(2): 1-11. https://doi.org/10.7149/ OPA.51.2.50026.

PANDEY, S. C. Y CATHER, S. (2015) "Close-range 3d imaging for documenting and monitoring dynamic deterioration processes in wall paintings", en ICOM CIDOC 2015: Documenting Diversity Collections, Catalogues \& Context.

PIERACCINI, M., GUIDI, G. Y ATZENI, C. (2001) "3D digitizing of cultural heritage", Journal of Cultural Heritage, 2(1): 63-70. https:// doi.org/10.1016/S1296-2074(01)01108-6.

RAHRIG, M., TORGE, M. Y ASSETS, C. (2019) "3D inspection of the restoration and conservation of stained glass windows using high resolution structured light scanning", en The International Archives of the Photogrammetry, Remote Sensing and Spatial Information Sciences, Volume XLII-2/W15, 2019: proceedings of the 27th CIPA International Symposium "Documenting the past for a better future", Ávila, España, 1-5 Septiembre 2019, 965-972. https:// doi.org/10.5194/isprs-archives-XLII-2-W15-965-2019

REMONDINO, F. (2011) "3D recording for cultural heritage", en Remote sensing for archaeological heritage management: proceedings of the 11th EAC Heritage Management Symposium, Reykjavík, Iceland, 25-27 Marzo 2010. Bruselas: Europae Archaeologia Consilium (EAC), 107-115.

RUIZ, J. F., SEBASTIÁN, M., QUESADA, E., PEREIRA, J., MAGUREGUI, M., FERNÁNDEZ, S., MARTÍNEZ, I., GIAKOUMAKI, A., MADARIAGA, J.M., PITARCH, A., LORENTE, J.C., DÓLERA, A. (2016) 4D • Arte Rupestre. Monografía. Dirección General de Bienes Culturales, Servicio de Patrimonio Histórico de la Región de Murcia.

RUIZ, J. F. Y PEREIRA, J. (2014) "The colours of rock art. Analysis of colour recording and communication systems in rock art research", Journal of Archaeological Science. Academic Press, 50(1): 338-349. https://doi.org/10.1016/j.jas.2014.06.023.

SANTOS MADRID, J. M. (2014) "El color en la reproducción fotográfica en proyectos de conservación", RevistaPh, 86(octubre), 102-123.

SAPIRSTEIN, P. (2018) "A high-precision photogrammetric recording system for small artifacts", Journal of Cultural Heritage, 31. https://doi.org/10.1016/j.culher.2017.10.011.

VANNESCHI, C., CAMILLO, M., AIELLO, E., BONCIANI, F., SALVINI,
R. (2019) "SFM-MVS photogrammetry for rockfall analysis and hazard assessment along the ancient roman via Flaminia road at the Furlo gorge (Italy)", ISPRS International Journal of GeoInformation, 8(8). https://doi.org/10.3390/ijgi8080325.

VERHOEVEN, G. (2018) "Resolving some spatial resolution issues - Part 1: Between line pairs and sampling distances", AARGnews, 57(October), 25-34. https://doi.org/10.5281/zenodo.1465017.

VERHOEVEN, G. (2019) "Resolving some spatial resolution issues - Part 2: When diffraction takes over", AARGnews, 59(October), 12-23.

\section{Autor/es}

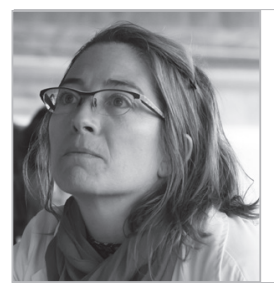

\section{Alba Fuentes-Porto}

afuentep@ull.edu.es

Servicio de Análisis y Documentación de Obras de Arte (SADOA-SEGAI), Grupo de investigación Ciencia y Patrimonio Universidad de La Laguna

Alba Fuentes-Porto es Diplomada en Conservación y Restauración de Bienes Culturales con especialidad de arqueología por la ECRBBCC de Galicia, Licenciada en Bellas Artes con especialidad en Restauración de Pintura por la Universidad Complutense de Madrid, y Máster en Conservación y Restauración de Bienes Culturales en la Universidad Politécnica de Valencia. En la actualidad es técnico en el Servicio de Análisis y Documentación de Obras de Arte (SADOA-SEGAI) de la Universidad de La Laguna; institución en la que desarrolla un doctorado en Arte y Humanidades ahondando en el campo del registro y documentación del Patrimonio.

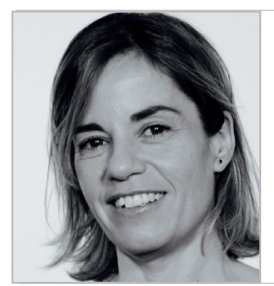

\section{Elisa Díaz-González}

ediazgon@ull.edu.es

Servicio de Análisis y Documentación de Obras de Arte (SADOA-SEGAl), Grupo de investigación Ciencia y Patrimonio Universidad de La Laguna

Elisa Díaz-González es profesora Contratada Doctora del Departamento de Bellas Artes de la Universidad de La Laguna. Imparte docencia en el Grado de Conservación y Restauración de Bienes Culturales y en el Máster en Uso y Gestión del Patrimonio Cultural. Entre 2008 y 2016 fue Profesora Asociada en la Universitat de Barcelona. Actualmente es responsable del Servicio de Análisis y Documentación de Obras de Arte (SADOA) perteneciente al Servicio General de Apoyo a la Investigación (SEGAI) de la Universidad de La Laguna. Licenciada en Bellas Artes con especialidad en Restauración Pictórica por la Universidad de Granada y diplomada en Conservación y Restauración de Bienes Culturales de Documento Gráfico por la Escola Superior de Conservació 
i Restauració de Béns Culturals de Catalunya. Doctora por la Universitat de Barcelona con una tesis sobre los libros ilustrados de Salvador Dalí y sus técnicas de ejecución. Miembro del grupo de investigación internacional Gruppo $130^{\circ}$ - The Group $130^{\circ}$, sobre las aplicaciones del papel coreano HANJI en la restauración de obra sobre papel y fotografía y coordinadora del grupo de investigación Ciencia y Patrimonio de la Universidad de La Laguna. Sus principales líneas de investigación se centran en los procesos fotográficos antiguos, los métodos para la inhibición de la corrosión de las tintas metaloácidas y en los sistemas de diagnosis por imagen.

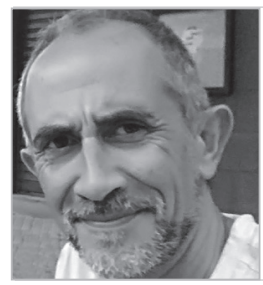

\section{Manuel Drago Díaz Alemán}

madradi@ull.edu.es

Servicio de Análisis y Documentación de Obras de Arte (SADOA-SEGAl), Grupo de investigación Ciencia y Patrimonio Universidad de La Laguna

Manuel Drago Díaz Alemán es Profesor Titular del Departamento de Bellas Artes de la Universidad de La Laguna y Director del Laboratorio de Diseño y Fabricación Digital de dicha Universidad. $\mathrm{Ha}$ sido Investigador principal en tres proyectos nacionales de carácter competitivo que determinan las líneas principales de su investigación que no son otras que las relativas a la implementación de las tecnologías gráficas avanzadas de diseño y la fabricación digital, en las Artes, el Diseño y la Conservación Bienes Culturales. Se ha especializado en impresión 3D, técnicas de escaneado tridimensional, fotogrametría, y el uso del modelado y animación 3D en el desarrollo de material didáctico de carácter científico.

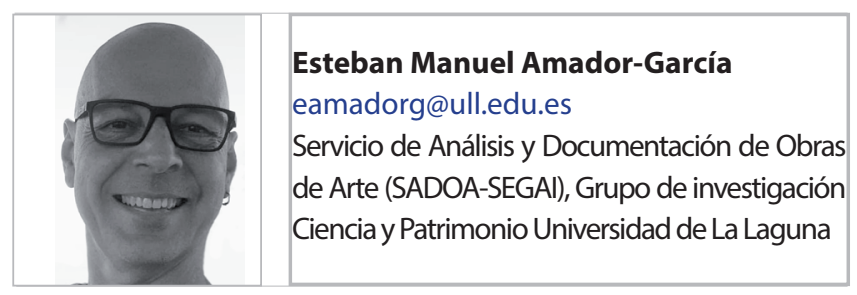

Doctor en Arte y Humanidades, Máster en Formación del Profesorado de Educación Secundaria y Bachillerato, Formación Profesional y Enseñanza de Idiomas, Graduado en Bellas Artes. Ha trabajado como técnico del Laboratorio de Diseño y Fabricación Digital de la Universidad de La Laguna (LFAB-SEGAI).

Especializado como generalista en la producción de imagen digital por medio de técnicas avanzadas de modelado y animación 3D y postproducción. Ha participado en numerosos proyectos de Innovación Educativa, así como en la producción de material didáctico y de divulgación de contenido científico. También acumula experiencia docente en el Grado de Bellas Artes y como profesor del catálogo formativo de la Fundación General de la Universidad de La Laguna. 